\title{
EL ENFOQUE STEAM Y SU IMPACTO EN LOS RESULTADOS ACADÉMICOS MEDIADOS POR LA CREENCIA DE CAPACIDAD O AUTOEFICACIA. REVISIÓN SISTEMÁTICA
}

\author{
Diego Bases-García \\ Universidad de León \\ dgarcb06@estudiantes.unileon.es \\ Jesús-Nicasio García-Sánchez \\ Universidad de León \\ jngars@unileon.es
}

Recepción Artículo: 13 septiembre 2021 Admisión Evaluación: 13 septiembre 2021 Informe Evaluador 1: 14 septiembre 2021 Informe Evaluador 2: 15 septiembre 2021 Aprobación Publicación: 17 septiembre 2021

\section{RESUMEN}

Se presenta una revisión sistemática de los estudios instruccionales publicados en los últimos cinco años sobre el impacto de los enfoques STEAM en los resultados académicos, mediados por la autoeficacia a través de las bases de datos WOS, Scopus y Frontiers identificando 6495 publicaciones, que, tras el proceso de cribado, idoneidad, se incluyen 8 estudios. Se siguen las cuatro fases de la revisión sistemática sistemática, comenzando con la selección del tema y el diseño del diagrama de términos de búsqueda, aplicación de criterios de inclusión y exclusión, análisis de la calidad de los estudios, y presentación de los resultados cualitativos y graficación del PRISMA. El análisis se centró en tres tipos de indicadores, un análisis general, un análisis de los componentes instruccionales y un análisis de las limitaciones del estudio. Se puede concluir que los enfoques STEAM inciden positivamente en el nivel de autoeficacia del alumnado y por consiguiente se consigue un incremento de los resultados de aprendizaje. Se discute el valor añadido del trabajo, sus implicaciones y aplicaciones, así como las perspectivas futuras y se concretan las conclusiones de la revisión sistemática.

Palabras claves: enfoques STEAMs; autoeficacia; programas instruccionales; revisión sistemática

\section{ABSTRACT}

The steam approach and its impact on academic outcomes mediated by ability or self-efficacy beliefs. A systematic review. We present a systematic review of the instructional studies published in the last five years on the impact of STEAM approaches on academic results, mediated by self-efficacy through the WOS, Scopus and Frontiers databases, identifying 6495 publications, which, after the screening process, suitability, 8 studies are included. The four of the systematic review, topic and diagram of search terms, inclusion and exclusion criteria, analysis of the quality of the studies, and qualitative results and contribution of the PRISMA are 


\section{EL ENFOQUE STEAM Y SU IMPACTO EN LOS RESULTTADOS ACADÉMICOS MEDIADOS POR LA CREENCIA DE CAPACIDAD O AUTOEFICACIA. REVISIÓN SISTEMÁTICA}

followed. The analysis focused on three types of indicators, a general analysis, an analysis of the instructional components, and an analysis of the limitations of the study. It can be concluded that STEAM approaches positively affect the level of self-efficacy of students and consequently an increase in learning results is achieved.The added value of the work, its implications and applications, as well as future prospects are discussed and the conclusions of the systematic review are specified.

Keywords: STEAMS approaches; self-efficacy; instructional programs; systematic review

\section{INTRODUCCIÓN}

En estos momentos, debido a los factores actuales originados por la COVID- 19, en los que toda la comunidad educativa se ha visto obligada a pasar de un modelo presencial y controlado a uno online, ha provocado una menor supervisión y guía por parte del profesorado a lo largo de todo el proceso de aprendizaje del alumno y por consiguiente, un descenso en los niveles de motivación, en la creencia de logro, y en los resultados de aprendizaje (López y Sanz, 2021).

La presentación de los contenidos en las distintas plataformas online juegan un papel fundamental, ya que, un entorno accesible, motivador y que permita al alumnado participar en su propio proceso de aprendizaje incide directamente en la superación de los objetivos educativos (Lin y Tsai, 2021).

A pesar de cómo se presenten los contenidos, existen una serie de asignaturas dentro del currículo que, dada la creencia de dificultad asociados a las mismas, presentan peores resultados en el aprendizaje y menores indicadores en los constructos de motivación y de autoeficacia, y son aquellas relacionadas con las ciencias, como por ejemplo, las matemáticas (Cox et al., 2021).

Dentro de las diferentes metodologías existentes, la metodología STEAM, en inglés Science, Tecnology, Engineering, Arts and Maths, es una de las más enfocadas a mejorar los resultados de aprendizaje. Se basa en el uso de prácticas experienciales en el aprendizaje del área de ciencias, utilizando diferentes herramientas, como las sesiones inmersivas de realidad virtual (en adelante iVR), los videojuegos, la robótica, las tablets, entre otros (Gomez y Ros, 2021).

En este ensayo, se va a revisar cómo la metodología STEAM y las diferentes prácticas tecnológicas influyen directamente en el nivel de creencia de logro del alumnado y en los resultados de aprendizaje. En primer lugar, se va a realizar una búsqueda en bases de datos para seleccionar artículos científicos. En segundo lugar, se analizarán en base a tres focos de análisis. Para finalizar, se analizarán los resultados.

En general los diferentes estudios que tratan esta temática se desarrollan mayoritariamente en centros educativos de China, Nueva Zelanda y Dinamarca, pero no en España, por lo que esta realidad justifica el presente estudio.

En función de esta situación de escasez de estudios en nuestro país, se plantea la siguiente pregunta de investigación: ¿Las prácticas de la metodología STEAM tiene un impacto positivo en la mejora de la creencia de logro del alumnado y por consiguiente se obtienen mejores resultados?

Para dar respuesta a esta pregunta, se van a analizar los artículos seleccionados en torno a tres focos: análisis general, análisis de la intervención y análisis de las limitaciones de los mismos. Para así poder responder al objetivo: analizar las variables que inciden de forma positiva en el alumnado y en su aprendizaje.

Es previsible que a través de esta revisión, se contribuya al aprendizaje de la metodología STEAM, de la autoeficacia y la relación existente entre ambas con los resultados de aprendizaje.

\section{Marco Teórico}

La aparición de los entornos virtuales de aprendizaje constituyó un gran paso para la integración de la tecnología en educación, ya que no se tratan solamente de un listado de páginas web en las que se presenta el contenido de una forma más atractiva, sino que son interfaces diseñadas para permitir la interacción del alumno con su propio conocimiento y con sus iguales, es decir, favoreciendo el aprendizaje colaborativo (Dillenburg et al., 2002). 
En esta línea, muchos estudios como el de Boorboor et al. (2013), investigan el impacto de los medios digitales en la motivación del alumnado y su aprendizaje, pero pocos estudian la relación existente entre autoeficacia, resultados en el aprendizaje y tecnología educativa.

Entendemos por autoeficacia la creencia de capacidad que tiene un individuo a la hora de enfrentarse a una tarea. Este concepto, en educación, es de gran importancia a la hora de desarrollar contenidos o actividades, ya que, si el alumno cree que no va a ser capaz de realizar una tarea, no lo logrará (Bandura, 2009).

En la guía de autoeficacia propuesta por dicho psicólogo, se hace especial hincapié al nivel de desafío, ya que, si no está acorde con la creencia de capacidad del individuo no se logrará. Por ello, es muy importante desarrollar actividades en línea con el nivel curricular del alumnado y atendiendo a sus capacidades, sino, por el contrario, no conseguirán superarlo (Bandura, 2009).

Por todo ello, la metodología STEAM juega un papel muy destacado para el aprendizaje, ya que no solo presenta contenidos de áreas más costosas para el alumnado de una manera más atractiva Bogner et al. (2020), sino que diseña toda una realidad experiencial utilizando los últimos avances en tecnología para que el alumnado, a través de la participación en su propio aprendizaje, consiga alcanzar los objetivos establecidos (Hsaio y Su, 2021).

\section{REVISIÓN SISTEMÁTICA}

\section{Metodología}

Para realizar la siguiente revisión sistemática, se siguió la metodología de cuatro fases según el modelo ilustrado por Miller et al. (2018); Scott et al. (2018). Este enfoque utiliza cuatro fases muy estructuradas. Por una parte, se inicia la revisión con la determinación del tema, y términos de búsqueda.

Figura 1

Diagrama de términos de búsqueda de AE, de educación y de metodología STEAM

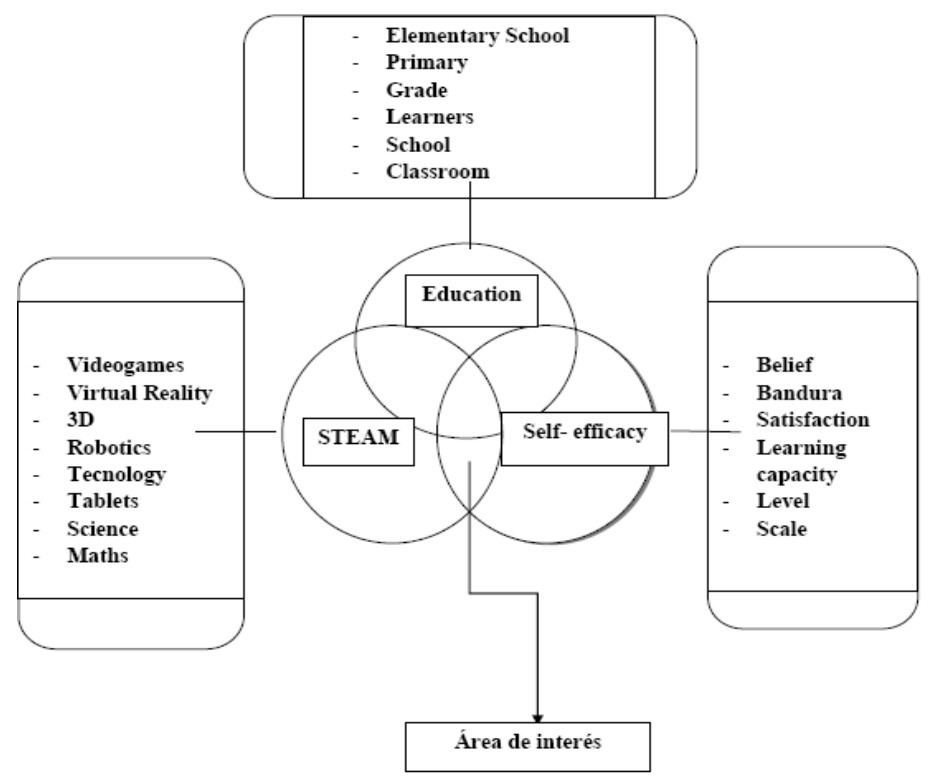




\section{EL ENFOQUE STEAM Y SU IMPACTO EN LOS RESULTADOS ACADÉMICOS MEDIADOS POR LA CREENCIA DE CAPACIDAD O AUTOEFICACIA. REVISIÓN SISTEMÁTICA}

En segundo lugar, se aplicaron los siguientes criterios de inclusión: i) publicaciones en el periodo de los últimos 5 años; ii) que se traten de estudios empíricos; iii) idioma en inglés y castellano; iv) que hagan referencia a STEAM y a programas educativos. Y de exclusión: i) Programas no validados; ii) artículos de reflexión u opinión; iii) otros idiomas que no sean ni inglés ni castellano; iv) artículos de otras áreas 0 ámbitos.

Por último, se realizó un análisis cualitativo de lo que aportan los estudios revisados con la presentación de Ios resultados graficados en el PRISMA (Moher et al., 2014). Al finalizar el proceso, se identificaron 8 artículos en las bases de datos Web Of Science, Sccopus y Frontiers.

Para su correcto análisis de la calidad de los estudios, se han elaborado tablas-tipo, teniendo en cuenta los siguientes indicadores: número de participantes, subgrupos, nivel educativo, foco, diseño, quién lo implementó, duración, instrumento, programa, procedimiento, estrategia, fiabilidad, limitaciones de la intervención, en los antecedentes, en la metodología, en los instrumentos, relevancia y limitaciones en los resultados.

2.2 Análisis general de las intervenciones

Comenzando con el análisis de los artículos seleccionados, se procede a una descripción general de los mismos, en la cual se han analizado aspectos como: número de participantes, subgrupos, nivel educativo, foco, diseño, quién lo implementó y duración (ver tabla 1).

Observando el número total de participantes, (en adelante N), podemos encontrar las primeras diferencias, ya que no existe una norma establecida en el número de participantes para la formación de las muestras. Los ejemplos más representativos de esta disparidad son el estudio de Bogner et al. (2020), compuesto por 928 alumnos de 16 países de la Unión Europea; y el realizado por Pande et al. (2021), en el cual se utilizó una muestra de 28 participantes de un curso de pregrado de Dinamarca.

La tónica general que rige los subgrupos (en adelante $n$ ), es que se dividen a su vez en dos, Grupo Experimental (en adelante GE) y Grupo Control (en adelante GC). El primer subgrupo, indica el número de participantes a los que se ha intervenido, mientras que el segundo, hace referencia a aquellos participantes que no han recibido el tratamiento. Analizando esta variable, se encuentra que 7 de los 8 estudios empíricos poseen ambos grupos. Es interesante el caso de un estudio que aplicó el tratamiento a todos los participantes, 928, pero, para facilitar su análisis posterior, utilizó solo una parte representativa de la muestra, un 10\% del total (Bogner et al., 2020). Este hecho resulta determinante para el análisis de los resultados, ya que si un estudio posee ambos grupos, va a poder comparar los datos obtenidos y así poder sacar conclusiones efectivas de las técnicas utilizadas.

Observando los niveles educativos en los que se han aplicado las diferentes intervenciones, engloban diferentes niveles educativos, como son Educación Primaria, Educación Secundaria, Bachillerato e incluso Estudios Universitarios, con lo que se puede analizar la aplicabilidad de la metodología STEAM en todo el marco educativo.

Centrándonos en la temática general, se observa que la metodología STEAM, sus componentes y el impacto en el nivel de autoeficacia del alumnado y los resultados en el aprendizaje, es la tónica en todos los estudios. Fruto del creciente interés por el uso de los últimos avances tecnológicos y su implementación en la educación, se están llevando a cabo multitud de estudios de comparación de medios, que investigan las ventajas del uso de la metodología STEAM frente a la enseñanza tradicional. Este hecho queda reflejado en investigaciones como las de Amor et al. (2020); Pande et al. (2021), que realizan una comparación entre el sistema de aprendizaje convencional y el realizado por medio de sesiones inmersivas de Realidad Virtual (en adelante iVR).

Continuando con el diseño de las investigaciones, decir que está marcado por el momento en el que se realizan las pruebas de evaluación y los subgrupos de análisis. En este punto, observamos que la mayoría de los estudios presentan el mismo diseño, GE frente a GC con medidas pre y post. Esto quiere decir, que a un grupo se le aplica la intervención y a otro no, realizando pruebas de medición antes y después del tratamiento y los resultados obtenidos de ambos grupos son comparados.

Sin embargo, encontramos diferencias en tres estudios. En primer lugar, el estudio de Lin y Tsai, (2021) que 
realizó las mediciones en tres momentos, pre, durante y post. En segundo lugar, el estudio de Bogner et al. (2020), que careció de GC. En último lugar, encontramos un estudio que aplicó la intervención en dos grupos experimentales frente a uno control, ya que en el primer GE se aplicó una intervención basada en videojuegos, en el segundo GE, juegos ordinarios y, en el tercero, el grupo control, se aplicaron sesiones magistrales (Wang y Zeng, 2020).

Pasando a la siguiente variable (quién implementó el tratamiento) encontramos que es una variable importante a tener en cuenta para el análisis posterior, ya que la metodología STEAM requiere de formación muy específica en el uso de nuevas tecnologías. La mayoría de los estudios se introdujeron en el aula por medio de maestros, profesores o investigadores especialistas en metodología STEAM, Io que nos asegura que el programa y su supervisión se desarrollaron de manera óptima, pudiendo solventar cualquier problema que hubiese surgido en su transcurso, ya fuese curricular o tecnológico.

Para finalizar, la duración de los diferentes estudios es muy variada. Está marcada principalmente por el contenido curricular que se quiso introducir en el aula. Por eso, tenemos intervenciones de un día de duración, como puede ser Wang y Zeng, (2020), en el que se introdujo un solo concepto científico, la refracción, mediante metodología STEAM, o de un año, en el cual se diseñó un Plan de Estudios que integró múltiples asignaturas y unidades didácticas. (Lin y Tsai, 2021). 


\section{EL ENFOQUE STEAM Y SU IMPACTO EN LOS RESULTADOS ACADÉMICOS MEDIADOS POR LA CREENCIA DE}

CAPACIDAD O AUTOEFICACIA. REVISIÓN SISTEMÁTICA

Tabla 1

Descripción general de los estudios analizados para esta revisión con metodología de la revisión sistemática

\begin{tabular}{|c|c|c|c|c|c|c|c|}
\hline Estudio & $N$ & $N$ & Curso & Foco & Diseño & Implementación & Duración \\
\hline $\begin{array}{l}\text { Bogner et } \\
\text { al. }(2020)\end{array}$ & $\begin{array}{c}928 \\
422 \\
\mathrm{H} \\
506 \\
\mathrm{M}\end{array}$ & $\mathrm{GE}=92$ & $\begin{array}{l}\text { EP, ESO, } \\
\text { Bach }\end{array}$ & $\begin{array}{l}\text { Creatividad y } \\
\text { modelo } \\
\text { pedagógico } \\
\text { STEM: } \\
\text { MI,MP y AE }\end{array}$ & $\begin{array}{c}\text { GE } \sin \mathrm{GC} \text { con } \\
\text { medidas pre } \mathrm{y} \\
\text { post }\end{array}$ & $\begin{array}{l}\text { Tutores de los } \\
\text { centros } \\
\text { seleccionados }\end{array}$ & $\begin{array}{l}1 \text { semana } \\
\text { a } 2 \text { años }\end{array}$ \\
\hline $\begin{array}{l}\text { Lin y Tsai } \\
(2021)\end{array}$ & $\begin{array}{l}114 \\
62 \\
\mathrm{H} \\
52 \\
\mathrm{M}\end{array}$ & $\begin{aligned} \mathrm{GE} & =57 \\
\mathrm{GC} & =57 .\end{aligned}$ & ESO & $\begin{array}{c}\text { Comparación } \\
\text { entre un Plan } \\
\text { de Estudios } \\
\text { STEAM y } \\
\text { uno ordinario }\end{array}$ & $\begin{array}{c}\text { GE frente GC } \\
\text { con medidas } \\
\text { pre, durante y } \\
\text { post. }\end{array}$ & $\begin{array}{l}\text { Profesores de } \\
\text { ciencias de dos } \\
\text { centros } \\
\text { educativos }\end{array}$ & $\begin{array}{c}\text { Curso } \\
\text { académico }\end{array}$ \\
\hline $\begin{array}{l}\text { Hsiao y Su } \\
\quad(2021)\end{array}$ & $\begin{array}{c}303 \\
\mathrm{H} \\
174 \\
\mathrm{M} \\
128\end{array}$ & $\begin{array}{c}\mathrm{GE}=303 \\
\mathrm{GC}=0\end{array}$ & EP & $\begin{array}{c}\text { Impacto del } \\
\text { Aprendizaje } \\
\text { experiencial } \\
\text { con sesiones } \\
\text { iVR en el } \\
\text { aprendizaje } \\
\text { AE y M }\end{array}$ & $\begin{array}{l}\text { GE } \sin \mathrm{GC} \text { con } \\
\text { medidas pre } \mathrm{y} \\
\text { post }\end{array}$ & $\begin{array}{l}\text { Investigadores } \\
\text { en tres centros } \\
\text { educativos }\end{array}$ & $\begin{array}{c}6 \text { sesiones } \\
\text { en } 1 \\
\text { semana }\end{array}$ \\
\hline $\begin{array}{l}\text { Wang y } \\
\text { Zeng } \\
(2020)\end{array}$ & 92 & $\begin{aligned} \mathrm{GE} & =31 \\
\mathrm{GE} & =30 \\
\mathrm{GC} & =31\end{aligned}$ & ESO & $\begin{array}{c}\text { Comparación } \\
\text { entre } \\
\text { Aprendizaje } \\
\text { basado en } \\
\text { videojuegos, } \\
\text { juegos } \\
\text { manipulativos } \\
\text { y sesión } \\
\text { magistral. }\end{array}$ & $\begin{array}{l}\text { Dos GE frente } \\
\text { GC con } \\
\text { medidas pre y } \\
\text { post. }\end{array}$ & $\begin{array}{l}\text { Maestro del } \\
\text { centro } \\
\text { educativo }\end{array}$ & $\begin{array}{c}2 \text { sesiones } \\
\text { de } 1 \mathrm{~h} \text { en } \\
\text { un día }\end{array}$ \\
\hline $\begin{array}{l}\text { Stiller y } \\
\text { Schworm } \\
(2019)\end{array}$ & $\begin{array}{c}39 \\
9 \mathrm{H} \\
30 \\
\mathrm{M}\end{array}$ & $\begin{array}{l}\mathrm{GE}=19 \\
\mathrm{GC}=20\end{array}$ & $\begin{array}{c}\text { Estudios } \\
\text { universitarios }\end{array}$ & $\begin{array}{c}\text { Efectos sobre } \\
\text { el aprendizaje } \\
\text { de conceptos } \\
\text { de Biología } \\
\text { en un idioma } \\
\text { extranjero } \\
\text { mediante } \\
\text { videojuegos } \\
\text { educativos o } \\
\text { hipertexto }\end{array}$ & $\begin{array}{l}\text { GE frente a GC } \\
\text { con medidas } \\
\text { pre y post }\end{array}$ & $\begin{array}{l}\text { Investigadores } \\
\text { en un campus } \\
\text { universitario }\end{array}$ & $\begin{array}{c}3 \text { sesiones } \\
\text { de } 1 \mathrm{~h} \text { en } \\
\text { un día }\end{array}$ \\
\hline $\begin{array}{c}\text { Fanchamps } \\
\text { et al. } \\
(2019)\end{array}$ & 62 & $\begin{array}{l}\mathrm{GE}=33 \\
\mathrm{GC}=29\end{array}$ & ESO & $\begin{array}{c}\text { Influencia del } \\
\text { Andamiaje o } \\
\text { de la } \\
\text { instrucción } \\
\text { directa en } \\
\text { Programación } \\
\text { Lego } \\
\text { Robotics: } \\
\text { habilidad } \\
\text { matemática y } \\
\text { AE }\end{array}$ & $\begin{array}{c}\text { GE frente a GC } \\
\text { con medidas } \\
\text { pre y post }\end{array}$ & $\begin{array}{c}\text { Maestros de } \\
\text { dos aulas de un } \\
\text { centro } \\
\text { educativo }\end{array}$ & $\begin{array}{c}9 \text { sesiones } \\
\text { de } 1 \mathrm{~h}\end{array}$ \\
\hline $\begin{array}{c}\text { Amor et al. } \\
(2020)\end{array}$ & $\begin{array}{l}125 \\
79 \\
\mathrm{H} \\
46 \\
\mathrm{M}\end{array}$ & $\begin{array}{l}\mathrm{GE}=32 \\
\mathrm{GE}=32 \\
\mathrm{GC}=31\end{array}$ & ESO & $\begin{array}{c}\text { iVR frente a } \\
\text { instrucción } \\
\text { directa y } \\
\text { lectura } \\
\text { ordinaria para } \\
\text { la } \\
\text { capacitación } \\
\text { en } \\
\text { emergencias } \\
\text { sísmicas: AE } \\
\text { y aprendizaje }\end{array}$ & $\begin{array}{l}2 \text { GE frente a } \\
\text { GC con } \\
\text { medidas pre y } \\
\text { post }\end{array}$ & $\begin{array}{c}\text { Investigadores } \\
\text { en un aula de } \\
\text { un centro } \\
\text { educativo }\end{array}$ & $\begin{array}{l}3 \text { sesiones } \\
\text { en un día }\end{array}$ \\
\hline $\begin{array}{c}\text { Pande et } \\
\text { al. (2021) }\end{array}$ & $\begin{array}{l}28 \\
14 \\
\mathrm{H} \\
14 \\
\mathrm{M}\end{array}$ & $\begin{array}{l}\mathrm{GE}=14 \\
\mathrm{GC}=14\end{array}$ & Bach & $\begin{array}{l}\text { Resultados de } \\
\text { aprendizaje } \\
\text { de sesiones de } \\
\text { iRV frente al } \\
\text { aprendizaje a } \\
\text { través de } \\
\text { video. }\end{array}$ & $\begin{array}{l}\text { GE frente a GC } \\
\text { con medidas } \\
\text { pre y post }\end{array}$ & $\begin{array}{c}\text { Profesor } \\
\text { universitario e } \\
\text { investigadores } \\
\text { en un aula de la } \\
\text { universidad }\end{array}$ & $\begin{array}{c}2 \text { meses y } \\
\text { medio }\end{array}$ \\
\hline
\end{tabular}




\section{Foco del proceso de intervenciones}

Continuando con el análisis de los artículos, se ha procedido a una revisión de las distintas intervenciones instruccionales, llevadas a cabo en cada uno de ellos, analizando las variables instrumento, programa, procedimiento, estrategia, fiabilidad y limitaciones de la intervención (ver tabla 2).

Para comenzar con el análisis de este foco, en primer lugar, se han identificado los diferentes instrumentos de medición utilizados. De manera generalizada, al tratarse de estudios que se realizan en centros educativos, se utilizan como mínimo dos instrumentos de medición, uno para evaluar los constructos psicológicos y, otro, para determinar el nivel de conocimiento adquirido con la intervención.

Comenzando con los instrumentos de análisis de constructos psicológicos, encontramos que en Fanchamp et al. (2020); Amor et al. (2020); Wang y Zeng (2020), la medición del constructo psicológico objeto de análisis de esta revisión, la autoeficacia, se ha llevado a cabo utilizado la Escala General de Autoeficacia de Bandura. Sin embargo, para poder implementarla correctamente se realizaron diferentes modificaciones de dicha escala para ajustar las cuestiones de análisis a las necesidades específicas de cada estudio (Bandura, 2009). Por otro lado, en el resto de investigaciones, se utilizaron cuestionarios de análisis múltiple, con el fin de medir varios constructos, como es el cuestionario utilizado en Hsiao y Su (2021) que consta de 47 ítems en los que se evaluó la autoeficacia, el conocimiento adquirido, la motivación, la satisfacción del alumnado y el efecto de la intervención.

Continuando con el análisis de los instrumentos, para poder evaluar los resultados de aprendizaje y la correcta asimilación de los contenidos, se han utilizado diferentes pruebas, como la encontrada en Pande et al. (2020) que aplica un cuestionario para evaluar el conocimiento adquirido en sesiones iVR que simulan las prácticas en un laboratorio de Biología.

Pasando a la variable 'programa', decir que, en prácticamente todas las investigaciones, se trabaja contenido curricular junto con instrumentos directamente relacionados con la metodología STEAM. Muestra de ello, es el estudio de Fanchamps et al. (2019), en el cual se realizan actividades para desarrollar la habilidad matemática mediante ejercicios con Lego Robotics, o en otro de los estudios, encontramos que se implementan 5 módulos de carácter cientííco relacionado con las matemáticas, la biología y la tecnología (Bogner et al., 2020).

El único programa que difiere del resto es el realizado por Amor et al. (2020), ya que utiliza las sesiones iVR para el aprendizaje experiencial en situaciones de emergencias sísmicas en un centro educativo de Nueva Zelanda.

Siguiendo con la siguiente variable, el procedimiento utilizado, encontramos que en seis de los ocho artícuIos, el aprendizaje experiencial basado en problemas (en adelante EBP) es el más utilizado. En este procedimiento instruccional se enfrenta al alumno a un problema y tiene que desarrollar estrategias para resolverlo. Es lógico el uso de este procedimiento, ya que el aprendizaje mediante experiencias es uno de los pilares básicos de la metodología STEAM, es decir, utilizar diferentes recursos mediante los cuales el alumno puede interactuar con el problema de primera mano y así resolverlo.

Un claro ejemplo es la investigación desarrollada por Stiller y Schworm, (2019), en la que a través de la interacción del alumno con los distintos niveles y desafíos de un videojuego educativo, se introduce una Unidad Didáctica del área Biología.

Aunque el EBP es el procedimiento mayoritario, no es el único, ya que encontramos en el estudio desarrollado por Lin y Tsai (2021) el uso de otro procedimiento instruccional, el denominado 5E. Este procedimiento esta desarrollado en cinco fases: compromiso, exploración, explicación, elaboración y evaluación, que se desarrollan a lo largo de toda la intervención.

Siguiendo con el análisis, encontramos la variable 'fidelidad', la cual es analizada a través de tres parámetros: medición, protocolo y control de la aplicación.

La medición nos informa del momento en el que se aplican los diferentes instrumentos para recoger datos. Podemos decir que casi todas las investigaciones utilizan como mínimo dos momentos, es decir, realizan una prueba inicial y una final, con el objetivo de recoger datos antes y después de aplicar la intervención y así comparar los resultados, es el caso de Fanchamps et al. (2019) y Wang y Zeng, (2020) entre otros. 


\section{EL ENFOQUE STEAM Y SU IMPACTO EN LOS RESULTTADOS ACADÉMICOS MEDIADOS POR LA CREENCIA DE CAPACIDAD O AUTOEFICACIA. REVISIÓN SISTEMÁTICA}

También encontramos estudios que aplican los instrumentos de medición en tres momentos, antes, durante y después, para poder incrementar la fidelidad de los datos obtenidos 0 analizar constructos psicológicos durante el proceso, como es el caso de Stiller y Schworm (2019), que utiliza la prueba QCM para analizar la ansiedad, el desafío, el interés y el éxito, factores determinantes de la carga cognitiva, tema principal del estudio.

En contraposición, el estudio de Hsiao y Su solo aplica los instrumentos de medición al finalizar la intervención, por tanto, no se puede analizar tan rigurosamente la variación de los niveles de motivación y autoeficacia en el alumnado como en los estudios anteriores (Hsiao y Su, 2021).

Hablando del protocolo, es decir, la lógica de la intervención, encontramos que todos los estudios poseen GE y GC, con el fin de medir los resultados obtenidos del GE y así poder compararlos y analizarlos con un grupo de las mismas características en el cual no se ha aplicado la intervención. Sin embargo, existen casos, como el estudio de Bogner et al. (2020), que solo poseen GE, ya que se aplica el programa a toda la muestra.

Para terminar con la fidelidad, se encuentra la aplicación del protocolo, es decir, el análisis de las tareas que realiza tanto el alumno como el entrenador, en este caso, investigador, durante el transcurso de la intervención. Cabe decir que los investigadores de los distintos estudios han realizado actividades de creación de entornos, tanto digitales como físicos, para favorecer el aprendizaje, de guía y supervisión del proceso. A su vez, los alumnos han realizado las tareas planteadas con la menor ayuda posible para no interferir en los resultados obtenidos.

Para concluir con el análisis de este foco, las limitaciones de la intervención es una variable importante a tener en cuenta. Algunas limitaciones se han desarrollado a lo largo de este apartado, como es el caso de Bogner et al. (2020) que carece de GE, o Hsiao y Su (2021) que solo realizan mediciones al finalizar la intervención.

Pero existen otras como son las encontradas en Lin y Tsai (2021), ya que realiza una intervención de 40 semanas y solo realiza tres momentos de medición, o Fanchamps et al. (2019) y Stiller y Schworm (2019), que no especifican las preguntas realizadas en los cuestionarios.

Para finalizar, la pandemia que estamos sufriendo puede alterar también las investigaciones realizadas y, un claro ejemplo, es la limitación encontrada en Pande et al. (2021), que tuvieron que modificar los integrantes tanto del GE y GC afectando al análisis posterior, ya que hubo un caso de Covid en uno de los grupos que afectó a varios miembros y, por consiguiente, no pudieron realizar todas las pruebas.

\section{Identificación de limitaciones}

Para finalizar con el estudio de los artículos revisados, se ha procedido a un análisis de las limitaciones de los artículos, donde se destacan variables como limitaciones en antecedentes, metodología, instrumento, relevancia y resultado (ver tabla 3 ).

Comenzando con las limitaciones en antecedentes, en este apartado se ha estudiado la antigüedad de las fuentes, si existe pregunta de investigación e hipótesis o si aparece clarificado el objetivo de la investigación.

En primer lugar, fijándonos en la antigüedad de las fuentes, se encontró que la mayoría de los estudios se justifican utilizando citas actuales, como máximo 5 años de antigüedad, esto es debido a la actualidad de las técnicas utilizadas, como pueden ser las sesiones iVR. 
Tabla 2

Análisis de los estudios revisados en torno al foco de indicadores de la intervención, incluyendo instrumento de evaluación, programa, procedimiento y estrategia, fidelidad y limitaciones de la intervención

\begin{tabular}{|c|c|c|c|c|c|c|c|c|c|}
\hline \multirow[t]{2}{*}{ Estudio } & \multicolumn{2}{|c|}{ Instrumento } & \multirow[t]{2}{*}{ Programa } & \multirow{2}{*}{\multicolumn{2}{|c|}{$\begin{array}{l}\text { Procedimiento/ } \\
\text { estrategia }\end{array}$}} & \multicolumn{3}{|c|}{ Fidelidad } & \multirow[t]{2}{*}{$\begin{array}{c}\text { Limitacion } \\
\text { es }\end{array}$} \\
\hline & Nombre & Constructo & & & & $\begin{array}{l}\text { Medici } \\
\text { ón }\end{array}$ & $\begin{array}{l}\text { Protoco } \\
\text { lo }\end{array}$ & $\begin{array}{l}\text { Control de la } \\
\text { aplicación }\end{array}$ & \\
\hline $\begin{array}{l}\text { Bogner et } \\
\text { al. }(2020)\end{array}$ & $\begin{array}{l}\text { Cuestionar } \\
\text { io con tres } \\
\text { subescalas }\end{array}$ & $\begin{array}{c}\text { Autoeficaci } \\
\text { a } \\
\text { Motivación } \\
\text { Creatividad }\end{array}$ & $\begin{array}{c}5 \text { módulos } \\
\text { académico } \\
\mathrm{s} \text { del área } \\
\text { de Ciencias }\end{array}$ & $5 \mathrm{E}$ & $\begin{array}{c}\text { Compromis } \\
\text { o } \\
\text { exploración } \\
\quad \\
\text { explicación, } \\
\text { elaboración } \\
\text { y } \\
\text { evaluación }\end{array}$ & $\begin{array}{l}\text { Pre y } \\
\text { post }\end{array}$ & $\begin{array}{l}\text { GE sin } \\
\text { GC }\end{array}$ & $\begin{array}{c}\text { Tutores: } \\
\text { supervisión } \\
\text { con escasa } \\
\text { participación }\end{array}$ & $\begin{array}{c}\text { Carece de } \\
\text { GC y no } \\
\text { especifica } \\
\text { duración }\end{array}$ \\
\hline $\begin{array}{l}\text { Lin y } \\
\text { Tsai } \\
(2021)\end{array}$ & $\begin{array}{c}\text { Kipsee } \\
\text { STML } \\
\text { Entrevista }\end{array}$ & $\begin{array}{c}\text { Conocimien } \\
\text { to } \\
y \\
\text { Autoeficaci } \\
\text { a } \\
\text { Motivación } \\
\text { Autoeficaci } \\
\text { a, } \\
\text { Motivación } \\
\text { y habilidad }\end{array}$ & $\begin{array}{l}\text { Plan de } \\
\text { Estudios } \\
\text { STEAM } \\
\text { aplicado en } \\
40 \text { semanas }\end{array}$ & $\begin{array}{c}\text { STEA } \\
M \\
\text { Viable }\end{array}$ & $\begin{array}{l}\text { Andamiaje, } \\
\text { tutoría, } \\
\text { atractivo, } \\
\text { modelado }\end{array}$ & $\begin{array}{c}\text { Pre, } \\
\text { durante } \\
\text { y post }\end{array}$ & $\begin{array}{c}2 \\
\text { grupos } \\
\text { con } 1 \\
\text { GC }\end{array}$ & $\begin{array}{l}\text { Tutor: } \\
\text { recursos, } \\
\text { guía } \\
\text { Alumno: } \\
\text { resolución }\end{array}$ & $\begin{array}{l}\text { Escaso } \\
\text { número de } \\
\text { pruebas } \\
\text { para un } \\
\text { periodo tan } \\
\text { extenso }\end{array}$ \\
\hline $\begin{array}{c}\text { Hsiao y } \\
\text { Su } \\
(2021)\end{array}$ & $\begin{array}{l}\text { Cuestionar } \\
\text { io de } 47 \\
\text { items }\end{array}$ & $\begin{array}{c}\text { Conocimien } \\
\text { to } \\
\text { Autoeficaci } \\
\text { a } \\
\text { Motivación } \\
\text { Satisfacción } \\
\text { Efecto }\end{array}$ & $\begin{array}{c}\text { Experienci } \\
\text { a, } \\
\text { Observació } \\
\mathrm{n}, \\
\text { Formación } \\
\mathrm{y} \\
\text { Actividade } \\
\text { s de } \\
\text { desafio }\end{array}$ & E.B.P & $\begin{array}{c}\text { Observació } \\
\text { n, } \\
\text { cooperación } \\
\text { y resolución } \\
\text { de } \\
\text { problemas }\end{array}$ & Post & $\begin{array}{c}2 \\
\text { grupos } \\
\text { con } 1 \\
\text { GC }\end{array}$ & $\begin{array}{c}\text { Investigador } \\
\text { es: creación } \\
\text { y } \\
\text { supervisión. } \\
\text { Alumnos: } \\
\text { resolución }\end{array}$ & $\begin{array}{c}\text { No detalla } \\
\text { las } \\
\text { actividades } \\
\text { del curso } \\
\text { de } \\
\text { formación }\end{array}$ \\
\hline $\begin{array}{l}\text { Wang y } \\
\text { Zeng } \\
(2020)\end{array}$ & $\begin{array}{c}\text { Escala de } \\
\text { Autoeficac } \\
\text { ia } \\
\text { Adaptada } \\
\\
\text { Cuestionar } \\
\text { io nacional }\end{array}$ & $\begin{array}{l}\text { Concepto } \\
\text { científico }\end{array}$ & $\begin{array}{l}\text { Concepto } \\
\text { científico } \\
\text { en tres } \\
\text { enfoques: } \\
\text { juego } \\
\text { digital, no } \\
\text { digital y } \\
\text { lectura } \\
\text { tradicional }\end{array}$ & E.B.P & $\begin{array}{c}\text { Gamificaci } \\
\text { ón, } \\
\text { Andamiaje } \\
\text { y Modelado }\end{array}$ & $\begin{array}{l}\text { Pre y } \\
\text { post }\end{array}$ & $\begin{array}{c}3 \\
\text { grupos } \\
\text { con } 2 \\
\text { GE y } 1 \\
\text { GC }\end{array}$ & $\begin{array}{c}\text { Maestro: lo } \\
\text { imparte en } \\
\text { los tres } \\
\text { grupos } \\
\text { Alumnos: } \\
\text { Observación } \\
\text { y realización }\end{array}$ & $\begin{array}{l}\text { No aparece } \\
\text { contenido } \\
\text { de la } \\
\text { sesión } \\
\text { explicativa }\end{array}$ \\
\hline $\begin{array}{l}\text { Stiller y } \\
\text { Schworm } \\
(2019)\end{array}$ & $\begin{array}{c}\text { Cuestionar } \\
\text { io Previo } \\
\text { QCM } \\
\text { durante }\end{array}$ & $\begin{array}{c}\text { Conocimien } \\
\text { to y } \\
\text { motivación } \\
\text { Ansiedad, } \\
\text { desafio, } \\
\text { interés y } \\
\text { éxito } \\
\text { Carga } \\
\text { Cognitiva }\end{array}$ & $\begin{array}{c}\text { Unidad } \\
\text { Didáctica } \\
\text { en un } \\
\text { idioma } \\
\text { extranjero } \\
\text { mediante } \\
\text { videojuego } \\
\text { s } \\
\text { educativos } \\
\text { e } \\
\text { hipertexto }\end{array}$ & E.B.P & $\begin{array}{l}\text { Gamificaci } \\
\text { ón, } \\
\text { modelado, }\end{array}$ & $\begin{array}{c}\text { Pre, } \\
\text { durante } \\
\text { y post }\end{array}$ & $\begin{array}{c}2 \\
\text { grupos } \\
\text { con } 1 \\
\text { GC }\end{array}$ & $\begin{array}{c}\text { Investigador } \\
\text { es: } \\
\text { instrucciones } \\
\text { previas } \\
\text { Alumno: } \\
\text { resolución } \\
\text { de } \\
\text { problemas }\end{array}$ & $\begin{array}{l}\text { No detalla } \\
\text { los } \\
\text { cuestionari } \\
\text { os }\end{array}$ \\
\hline $\begin{array}{l}\text { Fancham } \\
\text { ps et al. } \\
(2019)\end{array}$ & $\begin{array}{c}\text { Escala de } \\
\text { Autoeficac } \\
\text { ia } \\
\text { adaptada }\end{array}$ & $\begin{array}{c}\text { Autoeficaci } \\
\text { a }\end{array}$ & $\begin{array}{l}\text { Medición } \\
\text { previa, } \\
\text { intervenció } \\
\text { n robótica } \\
\text { y prueba } \\
\text { posterior. }\end{array}$ & E.B.P & $\begin{array}{c}\text { Andamiaje } \\
\mathrm{e} \\
\text { instrucción } \\
\text { directa }\end{array}$ & $\begin{array}{l}\text { Pre y } \\
\text { post }\end{array}$ & $\begin{array}{c}2 \\
\text { grupos } \\
\text { con } 1 \\
\text { GC }\end{array}$ & $\begin{array}{c}\text { Investigador: } \\
\text { formación } \\
\text { previa y } \\
\text { supervisión } \\
\text { del proceso } \\
\text { Alumno: } \\
\text { resolución } \\
\text { de las tareas } \\
\text { de } \\
\text { cuadrícula }\end{array}$ & $\begin{array}{c}\text { No detalla } \\
\text { cuestionari } \\
\text { o de } \mathrm{AE}\end{array}$ \\
\hline $\begin{array}{l}\text { Amor et } \\
\text { al. }(2020)\end{array}$ & $\begin{array}{c}\text { Escala de } \\
\text { Autoeficac } \\
\text { ia } \\
\text { Adaptada }\end{array}$ & $\begin{array}{l}\text { Conocimien } \\
\text { to de } \\
\text { seguridad }\end{array}$ & $\begin{array}{c}\text { Medición } \\
\text { pre y post a } \\
\text { un } \\
\text { Aprendizaj } \\
\text { e } \\
\text { experiencia } \\
\text { l en } \\
\text { situaciones } \\
\text { de } \\
\text { emergencia } \\
\text { sísmica }\end{array}$ & E.B.P & $\begin{array}{c}\text { Gamificaci } \\
\text { ón, } \\
\text { modelado e } \\
\text { integración } \\
\text { en } \\
\text { situaciones }\end{array}$ & $\begin{array}{l}\text { Pre y } \\
\text { post }\end{array}$ & $\begin{array}{c}3 \\
\text { grupos } \\
\text { con } 1 \\
\text { GC }\end{array}$ & $\begin{array}{c}\text { Investigador } \\
\text { es: } \\
\text { formación } \\
\text { previa, } \\
\text { maestros y } \\
\text { alumnos } \\
\text { Alumno: } \\
\text { resolución } \\
\text { del problema }\end{array}$ & $\begin{array}{c}\text { No aparece } \\
\text { impreso de } \\
\text { riesgos }\end{array}$ \\
\hline $\begin{array}{l}\text { Pande et } \\
\text { al. (2021) }\end{array}$ & $\begin{array}{l}\text { Cuestionar } \\
\text { io }\end{array}$ & $\begin{array}{l}\text { Conocimien } \\
\text { to }\end{array}$ & $\begin{array}{c}\text { Realizació } \\
\text { n de } \\
\text { experiment } \\
\text { os de } \\
\text { laboratorio } \\
\text { con iVR o } \\
\text { video }\end{array}$ & E.B.P & $\begin{array}{c}\text { Aprendizaje } \\
\text { experiencial } \\
y \\
\text { gamificació } \\
\mathrm{n}\end{array}$ & $\begin{array}{l}\text { Pre, } \\
\text { durante } \\
\text { y post }\end{array}$ & $\begin{array}{c}2 \\
\text { grupos } \\
\text { con } 1 \\
\text { GC }\end{array}$ & $\begin{array}{l}\text { Investigador } \\
\text { es: creación } \\
\text { de entorno } \\
\text { virtual y } \\
\text { seguimiento. } \\
\text { Alumnos: } \\
\text { resolución } \\
\text { de } \\
\text { problemas }\end{array}$ & $\begin{array}{c}\text { Los grupos } \\
\text { se tuvieron } \\
\text { que } \\
\text { reconfigur } \\
\text { ar por } \\
\text { medidas } \\
\text { COVID }\end{array}$ \\
\hline
\end{tabular}




\section{EL ENFOQUE STEAM Y SU IMPACTO EN LOS RESULTADOS ACADÉMICOS MEDIADOS POR LA CREENCIA DE CAPACIDAD O AUTOEFICACIA. REVISIÓN SISTEMÁTICA}

Sin embargo, al referirse a los constructos psicológicos como motivación o autoeficacia, encontramos que Fanchamps et al. (2020); Stiller y Schworm (2019) y Wang y Zen (2020) utilizan citas de más de 10 años de antigüedad; 0 más de 20 años (Hsiao y Su, 2021).

Con respecto a la pregunta de investigación y la hipótesis, se ha encontrado que algunos artículos tienen ambas y otros carecen de alguna. Muestra de esta limitación es el estudio de Hsiao y Su (2021), que presenta varias hipótesis, pero carece de pregunta de investigación 0, a la inversa, que presentan preguntas y no hipótesis (Wang y Zeng, 2021). Un caso a destacar es el estudio de Amor et al. (2021), en el que las preguntas de investigación aparecen antes de la aplicación de la intervención y las hipótesis no aparecen hasta después de los resultados, es decir, en la conclusión del estudio.

Sin embargo, pasando al objetivo de la investigación, decir que todos los estudios poseen al menos uno 0 varios. En la mayoría, aparecen reflejados en un apartado dedicado a ellos y numerados, salvo en Hsiao y Su (2021), en el que aparecen incluidos en un párrafo en mitad de la redacción de la justificación de la investigación.

Pasando a otra variable, la metodología utilizada, se ha tenido en cuenta el número de participantes y que los constructos analizados estén en sintonía con el estudio.

Sobre el número de los participantes, decir que cinco de los ocho estudios utilizaron tamaños de muestra que no permiten la generalización de los resultados, ejemplo de ello es el estudio de Stiller y Schorm, que utiliza una muestra de 39 participantes en su estudio, por tanto se tendría que repetir la investigación partiendo de los datos del mismo, pero con una muestra más grande (Stiller y Schorm, 2019).

Refiriéndose a los constructos analizados, solo encontramos un caso en el que este no se encuentra en sintonía con el estudio. Es el caso de Bogner et al. (2020) ya que analiza la motivación profesional en un grupo de participantes con edades que oscilan entre los 6 y los 17 años, mostrando errores en los resultados de los más jóvenes, debido a su corta edad, porque el empleo es una variable muy lejana a ellos.

Continuando con las limitaciones en instrumentos, decir que la mayoría de los estudios, muestran datos de fiabilidad y validez en sus análisis e incluso detallan el software o programa utilizados. Sin embargo, la falta de algunos datos, han producido errores en los instrumentos como en Wang y Zeng (2020) que solo recogieron datos cuantitativos, con lo cual, el análisis de la autoeficacia resulta incompleto.

Con respecto a la relevancia de los artículos, se observa que es mayoritariamente vertical, es decir, enfocada al aprendizaje de conocimientos curriculares útiles para cursos posteriores. Este hecho se debe a que se tratan de intervenciones realizadas en la escuela, con el fin último de conseguir que el aprendizaje sea más efectivo

Sin embargo, encontramos un estudio con relevancia horizontal, es decir, aportan aprendizajes para la vida. Es el estudio de Amor et al. (2020) que utiliza la tecnología de las sesiones iVR y el aprendizaje experiencial típico de la metodología STEAM para aprender a actuar en caso de emergencia sísmica.

Para finalizar, en el análisis de los resultados, observamos que, en casi todos los estudios, las intervenciones han tenido resultados positivos en la mayoría de los constructos analizados y en los resultados de aprendizaje, pudiendo constatar así que las intervenciones han tenido los resultados esperados. Por el contrario, encontramos dos estudios que ofrecen resultados no esperados, asociados principalmente a la dificultad de las pruebas realizadas por los infantes Pande et al. (2021) 0 debido al tiempo escaso de intervención Fanchamp et al. (2019) dando resultados positivos, pero por debajo de la significación.

\section{Resultados comparativos}

Los diferentes estudios aportados, gracias al procedimiento de la revisión de sistemática, demuestran que el aprendizaje experiencial a través de recursos tecnológicos en áreas STEAM, como las sesiones de iVR o los videojuegos educativos, consiguen incrementar el nivel de autoeficacia del alumnado y los resultados de aprendizaje frente a la instrucción directa o la sesión magistral.

En múltiples ocasiones, las Ciencias y, más en concreto, las matemáticas, han supuesto un problema para los alumnos, tanto de motivación, Autoeficacia y con peores resultados académicos. Por ello, dicha metodología 
ofrece un camino por el cual el alumnado, a través de entornos virtuales atractivos y controlados, videojuegos educativos o la programación de robots, consiguen disfrutar del aprendizaje (Fanchamps et al., 2019).

El aprendizaje experiencial, y especialmente, el modelo instruccional Experiencias Basadas en Problemas propio de la metodología STEAM, ha resultado ser un elemento motivador para la práctica del alumnado, ya que enfrentarse a un problema 0 aprendizaje y resolverlo a través de la gamificación y las experiencias virtuales ha aportado grandes beneficios en la adquisición de conocimientos frente al método tradicional (Stiller y Schowm, 2019).

Tabla 3

Análisis de los estudios revisados en torno al foco de limitaciones de los estudios de intervención, agrupando los indicadores en relación con los antecedentes, la metodología, los instrumentos de evaluación, la relevancia y los resultados

\begin{tabular}{|c|c|c|c|c|c|}
\hline Estudio & $\begin{array}{l}\text { Limitaciones en } \\
\text { antecedentes }\end{array}$ & Metodología & Instrumentos & Relevancia & Resultados \\
\hline $\begin{array}{l}\text { Bogner et } \\
\text { al. }(2020)\end{array}$ & $\begin{array}{l}\text { Las preguntas de } \\
\text { investigación no } \\
\text { aparecen destacadas. } \\
\text { La fundamentación se } \\
\text { centra en la creatividad } \\
\text { pero no en el resto de } \\
\text { constructos analizados. }\end{array}$ & $\begin{array}{c}\text { No se puede } \\
\text { generalizar ya que } \\
\text { utiliza una muestra } \\
\text { muy pequeña. } \\
\text { Un constructo, la } \\
\text { motivación laboral, no } \\
\text { está en sintonía con el } \\
\text { estudio. }\end{array}$ & $\begin{array}{l}\text { Aporta datos de } \\
\text { validación y } \\
\text { fiabilidad, pero no } \\
\text { define el tiempo } \\
\text { de aplicación del } \\
\text { programa. }\end{array}$ & $\begin{array}{l}\text { Relevancia } \\
\text { vertical }\end{array}$ & $\begin{array}{c}\text { De } 5 \text { constructos } \\
\text { analizados, solo } 2 \\
\text { presentan resultados } \\
\text { positivos }\end{array}$ \\
\hline $\begin{array}{l}\text { Lin y Tsai } \\
\quad(2021)\end{array}$ & $\begin{array}{c}\text { Investigaciones } \\
\text { anteriores no reflejaban } \\
\text { las prácticas del maestro. } \\
\text { No aporta antecedentes } \\
\text { de los constructos } \\
\text { analizados }\end{array}$ & $\begin{array}{l}\text { Estudio con un gran } \\
\text { número de } \\
\text { participantes pero solo } \\
\text { de un centro } \\
\text { educativo. } \\
\text { Las variables } \\
\text { analizadas no están } \\
\text { justificadas en } \\
\text { antecedentes. }\end{array}$ & $\begin{array}{l}\text { Los diferentes } \\
\text { instrumentos } \\
\text { aportan datos de } \\
\text { validación y } \\
\text { fiabilidad. }\end{array}$ & $\begin{array}{l}\text { De gran } \\
\text { relevancia } \\
\text { vertical } \\
\text { pero escasa } \\
\text { relevancia } \\
\text { horizontal }\end{array}$ & $\begin{array}{c}5 \text { de las } 6 \text { variables } \\
\text { mostraron un } \\
\text { incremento frente al } \\
\text { GC }\end{array}$ \\
\hline $\begin{array}{l}\text { Hsiao y Su } \\
\quad(2021)\end{array}$ & $\begin{array}{l}\text { Citas con } 20 \text { años de } \\
\text { antigüedad } \\
\text { Los objetivos no } \\
\text { aparecen remarcados } \\
\text { No aparece pregunta de } \\
\text { investigación }\end{array}$ & $\begin{array}{c}\text { La muestra es } \\
\text { representativa e } \\
\text { incluye participantes } \\
\text { de distintas escuelas }\end{array}$ & $\begin{array}{l}\text { Muestras datos } \\
\text { tanto de } \\
\text { validación y } \\
\text { fiabilidad } \\
\text { aportados por } \\
\text { software de } \\
\text { análisis de datos }\end{array}$ & $\begin{array}{l}\text { Relevancia } \\
\text { vertical }\end{array}$ & $\begin{array}{l}\text { Incremento en todos } \\
\text { los constructos } \\
\text { vinculado con la } \\
\text { familiarización en } \\
\text { simulaciones iVR. }\end{array}$ \\
\hline $\begin{array}{l}\text { Wang y } \\
\text { Zeng } \\
(2020)\end{array}$ & $\begin{array}{l}\text { La mayoría de las citas } \\
\text { tienen más de } 10 \text { años. } \\
\text { Carece de hipótesis }\end{array}$ & $\begin{array}{l}\text { El tamaño de la } \\
\text { muestra es } \\
\text { relativamente pequeño } \\
\text { y no permite la } \\
\text { generalización. }\end{array}$ & $\begin{array}{l}\text { Solo recogieron } \\
\text { datos cuantitativos } \\
\text { y no cualitativos. }\end{array}$ & $\begin{array}{l}\text { Relevancia } \\
\text { vertical }\end{array}$ & $\begin{array}{c}\text { Resultados idénticos } \\
\text { en los dos GE frente } \\
\text { al GC }\end{array}$ \\
\hline $\begin{array}{l}\text { Stiller y } \\
\text { Schworm } \\
(2019)\end{array}$ & $\begin{array}{l}\text { Las citas tienen más de } \\
\qquad 10 \text { años }\end{array}$ & $\begin{array}{l}\text { Muestra pequeña no } \\
\text { permite } \\
\text { generalización. } \\
\text { Corta duración del } \\
\text { tiempo de juego: } \\
\text { experiencia de } \\
\text { aprendizaje limitada }\end{array}$ & $\begin{array}{l}\text { Calificaciones } \\
\text { subjetivas en la } \\
\text { carga cognitiva ha } \\
\text { producido errores } \\
\text { en los datos de } \\
\text { análisis }\end{array}$ & $\begin{array}{l}\text { Relevancia } \\
\text { vertical }\end{array}$ & $\begin{array}{c}\text { Muestra incremento } \\
\text { en las variables pero } \\
\text { falta de datos } \\
\text { objetivos en la carga } \\
\text { cognitiva y } \\
\text { motivación }\end{array}$ \\
\hline $\begin{array}{l}\text { Fanchamps } \\
\text { et al. } \\
\text { (2019) }\end{array}$ & $\begin{array}{l}\text { La mayoría de las citas } \\
\text { tienen más de } 10 \text { años }\end{array}$ & $\begin{array}{l}\text { Muestra de pequeño } \\
\text { tamaño por tanto no } \\
\text { generalizable } \\
\text { Falta por investigar } \\
\text { diferentes variables } \\
\text { como: influencia del } \\
\text { maestro en la } \\
\text { instrucción, tipo de } \\
\text { instrucción... }\end{array}$ & $\begin{array}{l}\text { Aporta datos de } \\
\text { validez y } \\
\text { fiabilidad }\end{array}$ & $\begin{array}{l}\text { Relevancia } \\
\text { vertical }\end{array}$ & $\begin{array}{l}\text { Muestra resultados } \\
\text { positivos pero se } \\
\text { sitúan por debajo de } \\
\text { la significación }\end{array}$ \\
\hline $\begin{array}{l}\text { Amor et al. } \\
\quad(2020)\end{array}$ & $\begin{array}{l}\text { La mayoría de las citas } \\
\text { tienen más de } 10 \text { años. } \\
\text { Solo aportan } \\
\text { antecedentes de un solo } \\
\text { constructo } \\
\text { La hipótesis aparece en } \\
\text { las conclusiones del } \\
\text { estudio }\end{array}$ & $\begin{array}{c}\text { Muestra } \\
\text { representativa } \\
\text { Los constructos que } \\
\text { mide no están } \\
\text { justificados en el } \\
\text { marco teórico }\end{array}$ & $\begin{array}{c}\text { No aporta datos de } \\
\text { validez y } \\
\text { fiabilidad de los } \\
\text { datos analizados }\end{array}$ & $\begin{array}{l}\text { Relevancia } \\
\text { Horizontal }\end{array}$ & $\begin{array}{l}\text { Diferencias no } \\
\text { significativas en } 2 \text { de } \\
\text { las tres variables en } \\
\text { los } 2 \mathrm{GE} \text { frente a GC }\end{array}$ \\
\hline $\begin{array}{l}\text { Pande et } \\
\text { al. (2021) }\end{array}$ & $\begin{array}{l}\text { El estudio muestra } \\
\text { pregunta de investigación } \\
\text { pero no hipótesis. }\end{array}$ & $\begin{array}{c}\text { Muestra poco } \\
\text { representativa para su } \\
\text { generalización. } \\
\text { Los constructos } \\
\text { analizados no figuran } \\
\text { en la justificación del } \\
\text { estudio }\end{array}$ & $\begin{array}{l}\text { Hubo que } \\
\text { discriminar un } \\
\text { grupo por lo que } \\
\text { la fiabilidad del } \\
\text { instrumento se } \\
\text { puede ver } \\
\text { comprometida }\end{array}$ & $\begin{array}{l}\text { Relevancia } \\
\text { vertical }\end{array}$ & $\begin{array}{c}\text { La dificultad de la } \\
\text { prueba ha afectado } \\
\text { significativamente al } \\
\text { resultado }\end{array}$ \\
\hline
\end{tabular}

International Journal of Developmental and Educational Psychology 


\section{EL ENFOQUE STEAM Y SU IMPACTO EN LOS RESULTADOS ACADÉMICOS MEDIADOS POR LA CREENCIA DE CAPACIDAD O AUTOEFICACIA. REVISIÓN SISTEMÁTICA}

Aunque los resultados de los distintos estudios no permiten la generalización debido al tamaño de las muestras, reflejan que al aplicar el modelo, constructos como la satisfacción, el interés y los efectos en el propio aprendizaje se incrementan del mismo modo que la autoeficacia.

Este hecho, que parece revelador, tiene una gran limitación a la hora de implementarlo, por un lado, el coste material, y por otro, la formación del profesorado, ya que, para poder implementarlo de manera generalizada, tendrían que recibir gran cantidad formación en programación para adaptar el contenido curricular a los entornos digitales.

A pesar de estos inconvenientes, la educación cada vez está más ligada a las tecnologías. Hace años sería impensable el uso de tablets en los centros y hoy es una realidad, por tanto, aunque hoy sea una realidad lejana, mañana será un hecho.

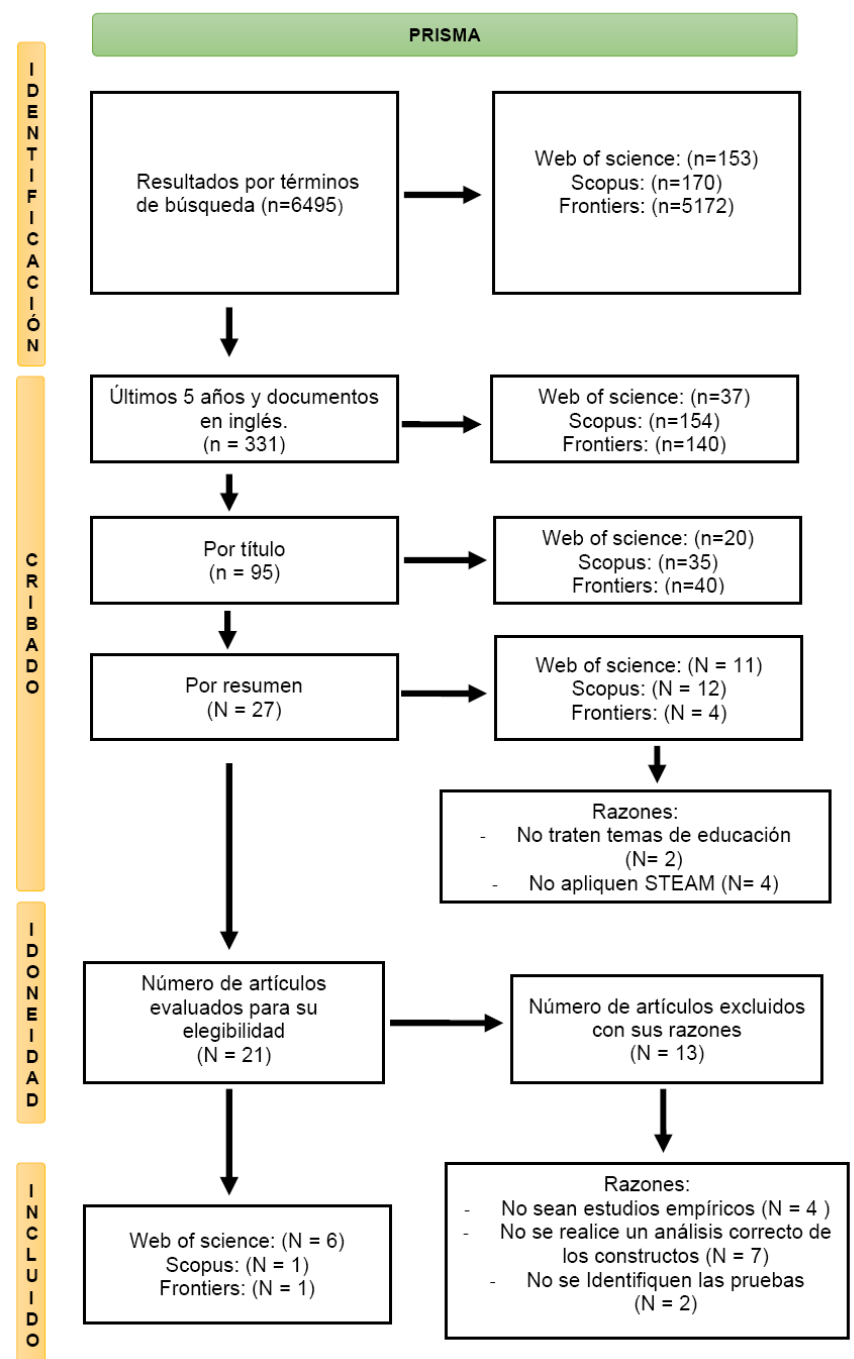




\section{DISCUSIÓN Y CONCLUSIONES}

Gracias al trabajo desarrollado a través de las diferentes fases de la presente revisión: selección de términos, búsqueda de artículos en diferentes bases de datos, establecimiento de criterios de inclusión e inclusión, lectura y análisis de los estudios *localizados, considero que este proceso me ha permitido formarme y desarrollar una mirada crítica para posteriores estudios, revisiones sistemáticas e incluso oposiciones.

Por otro lado, tras realizar el análisis y comparación de los resultados, considero que tanto la autoeficacia del alumnado como el aprendizaje experiencial, ya sea a través de medios informáticos o tradicionales, son elementos clave para poder desarrollar correctamente mi labor como futuro docente (Amor et al., 2020).

La creencia en la propia capacidad o autoeficacia resulta fundamental a la hora de realizar con éxito una actividad. Por ello, en todos los estudios analizados, presentar los contenidos de una manera atractiva y utilizar técnicas que inspiren al alumnado, han resultado fundamentales para que los alumnos, independientemente de la dificultad, consideren que sean capaces realizar las distintas actividades y así poder alcanzar los resultados de aprendizaje deseados (Bogner et al., 2020).

Sin embargo, existen elementos a mejorar para conseguir incrementar los resultados de estas intervenciones, los más destacables son tener en cuenta variables como la complejidad de la tarea, los recursos de los centros educativos y el coste de la tecnología. Ya que, por desgracia, a día de hoy, las herramientas necesarias para poder extrapolar a un aula ordinaria las intervenciones realizadas por Pande et al. (2021); Amor et al. (2020) y Hsiao y Su (2021), basadas en sesiones iVR, requieren de una gran inversión económica que a día de hoy la educación pública no puede asumir.

A pesar de los resultados obtenidos a lo largo de este proceso, la mayor limitación de la presente revisión es el tiempo, ya que para realizar una correcta revisión sistemática se necesitarían muchos meses y abarcaría un mayor número de artículos, sin embargo, se han seguido los 4 pasos establecidos en el procedimiento y he aprendido cómo hacerlo.

Para concluir, y volviendo al tema objeto de estudio, considero que las herramientas propias de la metodología STEAM, tales como la robótica o las sesiones iVR, no solo deberían aplicarse al área de ciencias, ya que cada alumno al tener sus propias concepciones de éxito, pueden no coincidir con las de la mayoría, por lo que se debería investigar sus efectos en todas las asignaturas del currículo académico.

\section{REFERENCIAS BIBLIOGRÁFICAS}

Amalia, R., Santosa, E. and Zaiyar, M. (2021). Android - Based Learning Enviroment to enhance creative thinking mathematics and Self-Eficaccy. Journal of Physics.(1764), 1- 12. https://doi.org/10.1088/17426596/1764/1/012133

*Amor, R., Cabrera-Guerrero, C., Feng, Z., Gonzalez, V. and Mutch, C. and (2020). Instructional mechanisms in immersive virtual reality serious games: Earthquake emergency training for children. Journal of computer assited Learning Wiley, (37), 542 - 556. https://doi.org/10.1111/jcal.12507

Bandura, A. (2009). Social cognitive theory in cultural context. Journal of Applied Psychology: An International Review, 51, 269-290.

Bandura, A. (2009). Social cognitive theory: An agentic perspective. Annual Review of Psychology, 52, 1-26.

Batten, J., Clarke, P., Damas, D., Davis, D., Potvin, G., Ross, M. and Zahedi, L. and (2021). Gamification in education: a mixed methods study of gender on computer science students' academic performance and identity development. Journal of Computing in Higher Education. https://doi.org/10.1007/s12528-021- 09271-5

${ }^{*}$ Bogner, F., Conradty,C. and Sotiriu, S. (2020). How creativity in STEAM Modules Intervenes with Selff- Efficacy and Motivation. Education Sciences, 3(70), 1-15. https://doi.org/10.3390/educsci10030070

Boorboor, B., Jafari, F. and Zarrabian, F. (2013). The study of utilization of information technology (IT) in students' scholarly achievement in smart and ordinary schools. Advances in Environmental Biology, 7(8) 1434 - 1442.

Cox, C., Fong, C., Kremer, K. and Lawson, C. (2021). Expectancy- value profiles in math and science: A person 


\section{EL ENFOQUE STEAM Y SU IMPACTO EN LOS RESULTADOS ACADÉMICOS MEDIADOS POR LA CREENCIA DE CAPACIDAD O AUTOEFICACIA. REVISIÓN SISTEMÁTICA}

- centered approach to cross - domains motivation with academic and STEAM - related outcomes. Contemporary Educational Psychology, 65 (1011962). https://doi.org/10.1016/j.cedpsych.2021.101962

Dillenbourg, P., Schneider, D. and Synteta, P. (2002). Virtual Learning Environments. 3rd Hellenic Conference "Information \& Communication Technologies in Education. 2002, Rhodes, Greece. pp.3-18.

*Fanchamps, N., Hennissen, P., Slangen, L. and Specht, M. The influence of SRA programming on algorithmic thinking and self efficacy using Lego robotics in two types of instruction. International Journal of Technology and Design Education 31, 203 - 222. https://doi.org/10.1007/s10798-019-09559-9

Gomez, C. and Ros, G. (2021). Transdisciplinary integration and its implementation in primary education through two STEAM projects (La integración transdisciplinar y su aplicación en Educación Primaria a través de dos proyectos STEAM). Journal for the study of education and development. https://doi.org/10.1080/02103702.2021.1925474

*Hsiao, P. and Su, C. (2019). A study on the Impact of STEAM Education for Sustainable Development Courses and its Effects on Student Motivation and Learning. Sustainability 2021, (13), 2 - 24. https://doi.org/10.3390/su13073772

Jepsen, M., Mojsoska, B., Moellen M., Pande, P., Sorensen, A. and Thit, A. (2021). Long-term effectiveness of immersive VR simulations in undergraduate science learning: lessons from a media-comparison study. Research in Learning Technology, 29(2482). http://dx.doi.org/10.25304/rlt.v29.2482

*Lin, C. and Tsai, C. (2021). The effect of a Pedagogical STEAM Model on Students Project Competence and Learning Motivation. Journal of Science Education and Technology 30(1), 112 - 124. https://doi.org/10.1007/s10956-020-09885-x

Lopez, E. y Sanz. (2021). Consecuencias pedagógicas de enseñanza básica derivadas de la COVID- 19. Una reflexión en torno a los grandes olvidados de la pandemia. Revista Universitaria, 33 (2), 149- 166. https://doi.org/10.14201/teri.245471

Miller, D., Mctigue, E., Scott, C. and Washburn,C (2018). The what, when, and how of preservice teachers and literacy across the disciplines: A systematic literature review of nearly 50 years of research. Elsevier, 73(July 2018) 1-13. https://doi.org/10.1016/j.tate.2018.03.010

Moher, D., Liberati, A., Tetzlaff, J., Altman, D. G., The PRISMA Group (2009). Preferred Reporting Items for Systematic Reviews and Meta-Analyses: The PRISMA Statement. PLOS Med 6(7): e1000097. https://doi.org/10.1371/journal.pmed1000097

Moher, D., Liberati, A., Tetzlaff, J., Altman, D. G., The PRISMA Group (2014). Ítems de referencia para publicar Revisiones Sistemáticas y Metaanálisis: La Declaración PRISMA. Rev Esp Nutr Hum Diet.; 18(3), 172-181. prisma-statement.org

*Pande, P., Jepsen, P., Moeller, M., Mojsoska, B., Sorensen, A. and Thit, A. (2021). Long-term effectiveness of immersive VR simulations in undergraduate science learning: lessons from a media- comparison study. Asociation for Learning Technology 29. http://dx.doi.org/10.25304/rlt.v29.2482

Qashou, A. (2020). Influencing factors in M-Learning adoption in higher education. Education and information Tecnologies, (26) 1755 - 1785. https://doi.org/10.1007/s10639-020-10323-z

*Schworm, S. and Stiller, K. (2019). Game- Based Learning of the Structure and Functioning of Body Cells in a Foreign Languaje: Effects on Motivation, Cognitive Load, and Performance. Frontiers in Education 4(18). https://doi.org/10.3389/feduc.2019.00018

*Wang, M. and Zheng, X. (2020). Using Game - Based Learning to Support Learning Science: A study with Middle School Students. Asia-Pacific Edu Res 30(2), 167-176. https://doi.org/10.1007/s40299-020-00523-z 